Engineering \& Technology | Žilvinas Svigaris

\section{Rethinking the reverse side of Artificial Intelligence}

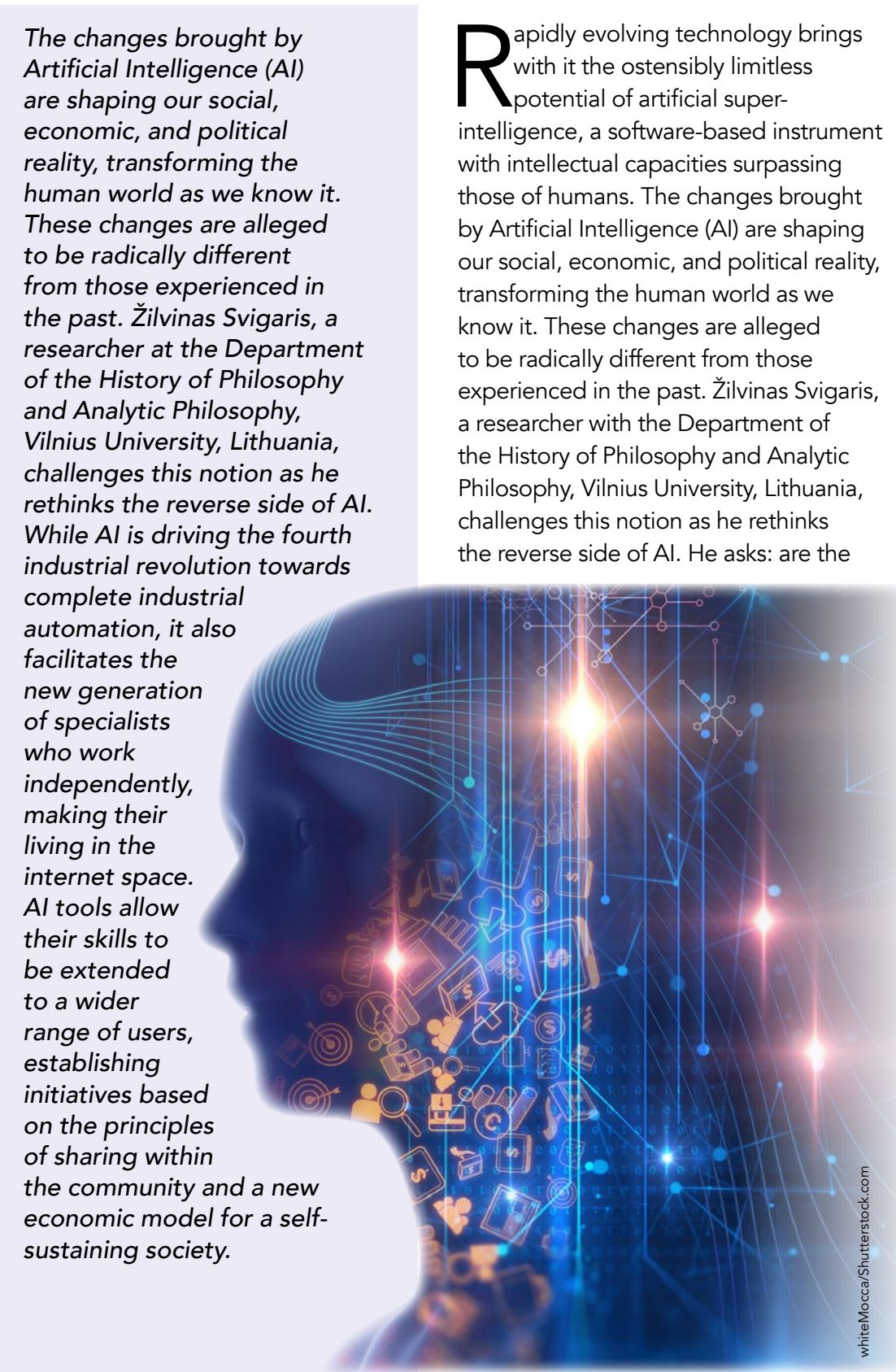

changes brought by Al really radically different? Moreover, what underpins can it not change? be different. Past technological advancements have already shown us that change can evolve in ways that could not have been predicted at their instigation. We live in a technological civilisation that appears to offer unlimited opportunities, but Svigaris warns that it also 'hides a spectre of the decline, if not a complete dismissal, of free, autonomous, and responsible

ARTIFICIAL INTELLIGENCE -

AN IMPROVED INSTRUMENT

Alis a manifestation of mathematical

concepts. An Al system does not

it discovers, or what its discoveries

mean. Its intelligence is quantitative

and, ultimately, digital. Humans are

not limited to deductive quantitative

thinking, but are capable of qualitative

and contemplative thinking, intertwined

with experience and human values.

Nevertheless, Al has infiltrated everyday life through a variety of tools. Not only is the living world changing, but human habits and identity are also shifting.

Almost everything concerning memory

and knowledge has transferred to

electronic databases, our orientation

around cities now comes in the form of

virtual maps, language skills emerge as

multilingual applications, and our daily social portals.
The Al systems that are shaping society, however, are not governmental organisations, but onl gearch portals, systems collecting and analysing user. We already remember less about ourselves than those systems belonging to companies such as Google,

Facebook, and Amazon, that monitor and keep track of our interests: what we ate, what we bought, what TV show we watched, where and when we travelled and many other things.

Svigaris observes that Al has become an improved instrument. Humans previously involved in the development process of creating their instruments are becoming obsenvers in the developm of modern technology. With this, Al of its creming a construct independent only creating technology but is also maintaining it This alters the state of what was previously called an instrument. Now, Al can exceed the abilities of its creator, making them dependent on its 'intelligence'. Every step of the global race towards the exponential

capabilities of Al repositions its creator's functions and values, both in terms

time.

\section{KNOWLEDGE}

Digtal technology is extending the possible for unlimited amounts of information to be

Consciousness is not a static phenomenon, but rather the ability to question different ways and change the very understanding of the living world.

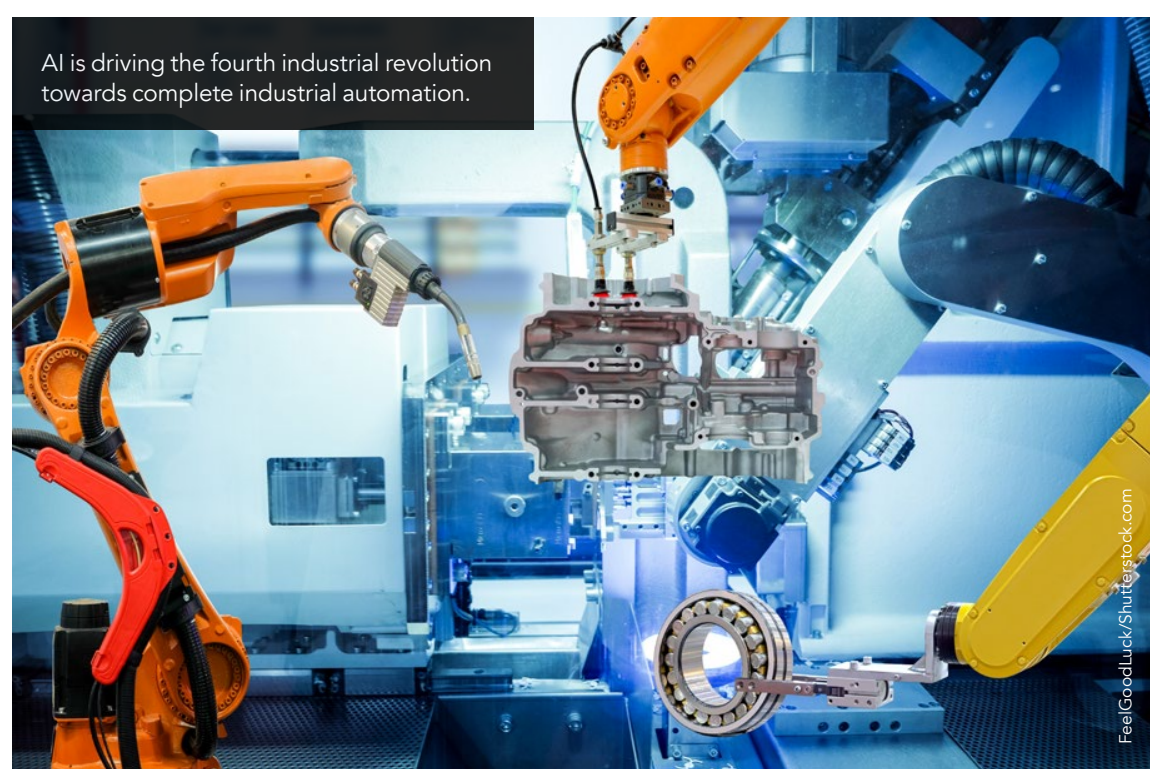

This accessibility opens avenues for global seff-education systems with discoveries, published journals, and online articles, accessible at the touch of a button. It is important to realise, however, that $\mathrm{Al}$ is ethically indifferent. nowledge is in flux - it is no longe permanent, nor is it truth or lie.

\section{INDUSTRIAL AUTOMATION} Al is driving the fourth industrial revolution This industrial transformation differs greatly from earlier industrial revolution. Where previously the development new instruments created new jobs

stored and accessed quickly. This changes the human relationship with knowledge. Historically, humanity has viewed knowledge as a great treasure. Digitalisation is opening up accessibility of information to the point of saturation, and as a result knowledge is losing ins exceptional value and elite status. Knowledge is being shared, making it an intermediate, rather than a final, state of thinking. Consequently, the instrumentality of knowledge is changing significantly. It is no longer static, binding, and possessing. Instead, knowledge has Kecome liberating, dynamic and alive. Knowledge is now intend
rather than accumulation. and specialisations, Al does not create new jobs; instead, it renders human abilities obsolete, causing humans to be redundant. We therefore face a glob quantitative manipulation supporting power among the few, or it can focus instrument for balancing human ingenuit in a qualitative environment.

Svigaris explains how this parting of the ways offers us a choice. Either we continue with the current mode of $\mathrm{Al}$, with its indifferent ethics concerning both humans and the environment, and disappear, or we invite Al as an ally to reestablish harmonious human life within a qualitative environment. The latter option awareness, the rejection of consumerism, and the promotion of sharing in our common humanity. While this trend strengthen the neo-liberal ide etther quantitative manipultion supporing appears to be emerging aided by global might be considered disruptive to global 
participate in the bottom-up creation of projects that can spread from community to community. Furthermore, environments, together with their biodiversity and the exchange of needs of other communities.

TRANSFORMATION OF EDUCATION The Western education system has a propensity for the technical, narrow, standardised training of specialists, effectively creating human instruments with specific qualifications. The implementation of Al automation is likely to push those with a narrow specialisation out of their working place. It may be time to rethink the need to train narrow-profile specialists, as before long these human instruments will no longer be required in many areas. At the same time there is a need for independent, conscious from instrumental ontoly Suigris discusses how'consciousness is not static phenomenon, but rather the ability to question different ways and change the very understanding of the living world'. It is therefore important to educate individuals who
can orientate We can "re-t

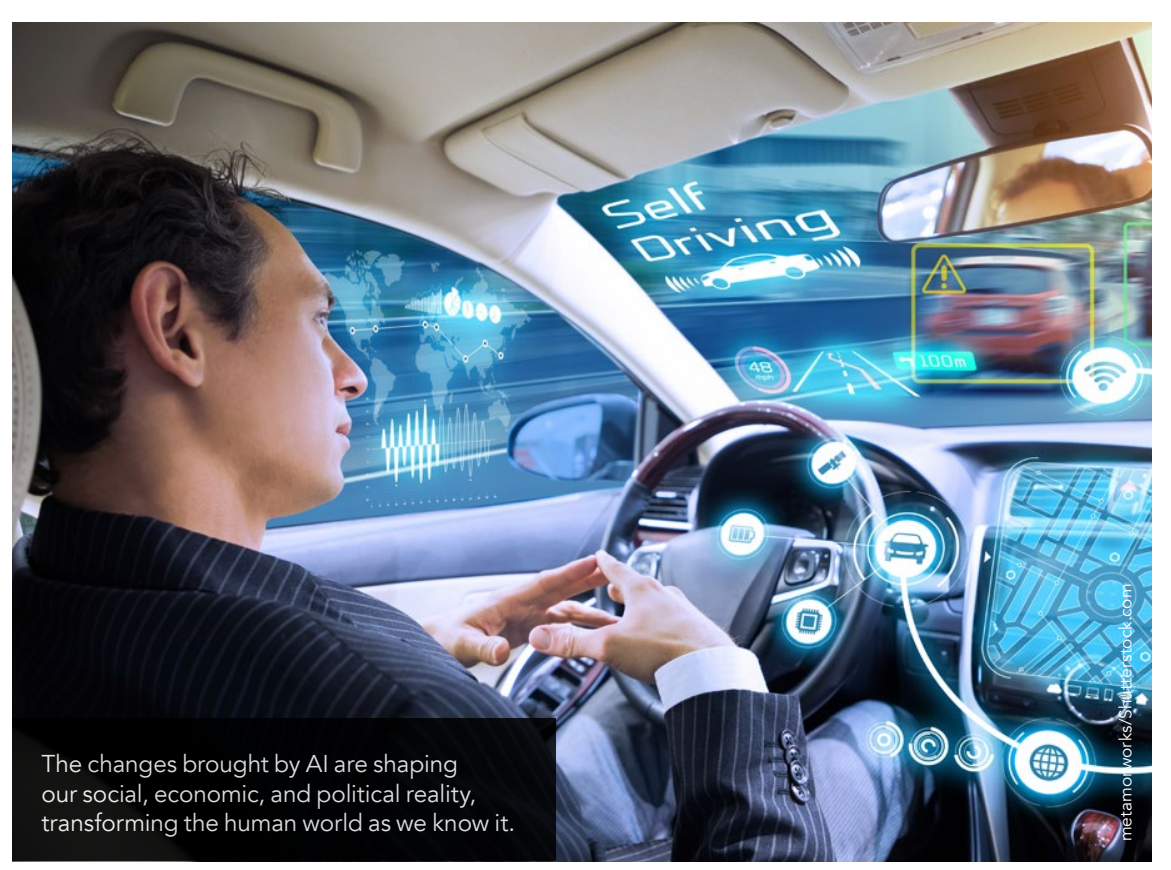
SELF-SUSTAINING SOCIETY The new generation of specialists work the internet space. Al their living in skills to be extended to a wider range of users. This expansion of sharing

but exploring new ways to share it. There are many examples of sharing initiatives, such as Amazon, where and Uber, where people can register and start earning as a driver. These being an indifferent and destructive force sharing within the themselves and and become a qualitative value partner. is changing as a harmoniously

adapt in a changing context with others goods, services and knowledge, is

and the environment. The concept of the gradually decentralising the global specialty requires different qualities to become adaptable, independ
open up to the living world. economy, ousting centralised own and dependency. Enterprises no
longer focus on accumulating property, wellbeing within its environment. This self-sustaining society brings with it a new economic model grounded on

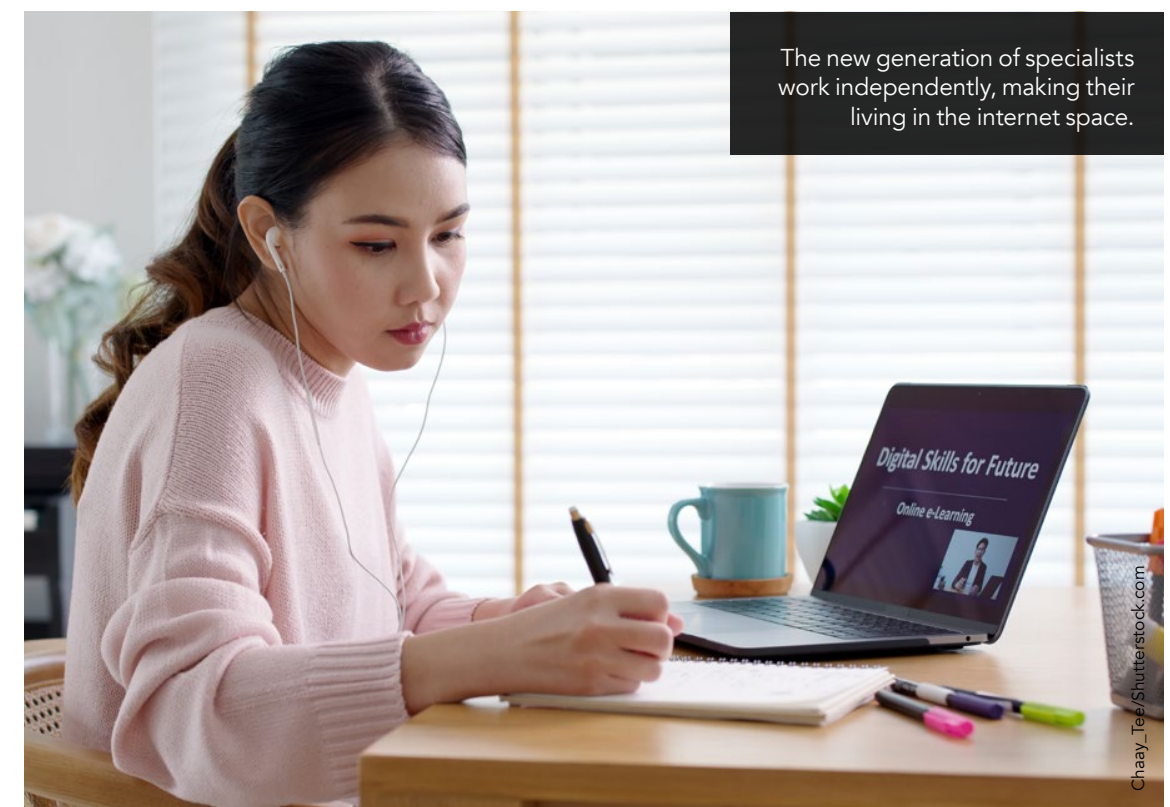
based on the principles of

\section{THE ONTOLOGICAL SHIFT OF AI} Our human world and our qualitative Our human world and our qualitative
environment are dependent on one another. We can 're-think' Al so that it can stop being an indifferent and destructive force and become a qualitative value partner with the ability to tell us which areas of the hat species are endangered. Svigaris concludes that this ontological shift of Al offers a 're-understanding' that can change our educational requirements. Moreover, it can transform the hum trapped in a world of specialised technical constructs, into a human who world that forms our home.

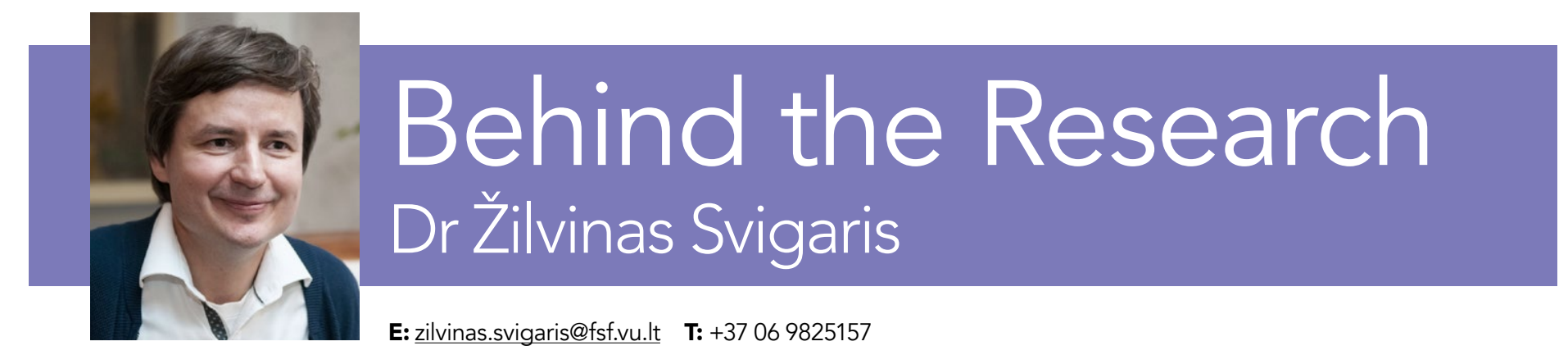

Research Objectives

Žilvinas Svigaris explores philosophical hermeneutics, ontology, phenomenology, and comparatives.

\section{Detail}

\section{Address}

Žilvinas Svigaris, 3T, Sausio 13-os, 2, Vilnius

Bio

Żivinas Svigaris graduated from Kaunas University of Technology in 1996 with a BSc in Computer Science and Programming and Economics. He completed a Masters in Economics at the same institute in 1998, and a Philosophy Masters in 2008 at Vilnius University. Žilvinas Svigaris defended his thesis in the field of Humanities Philosophy at
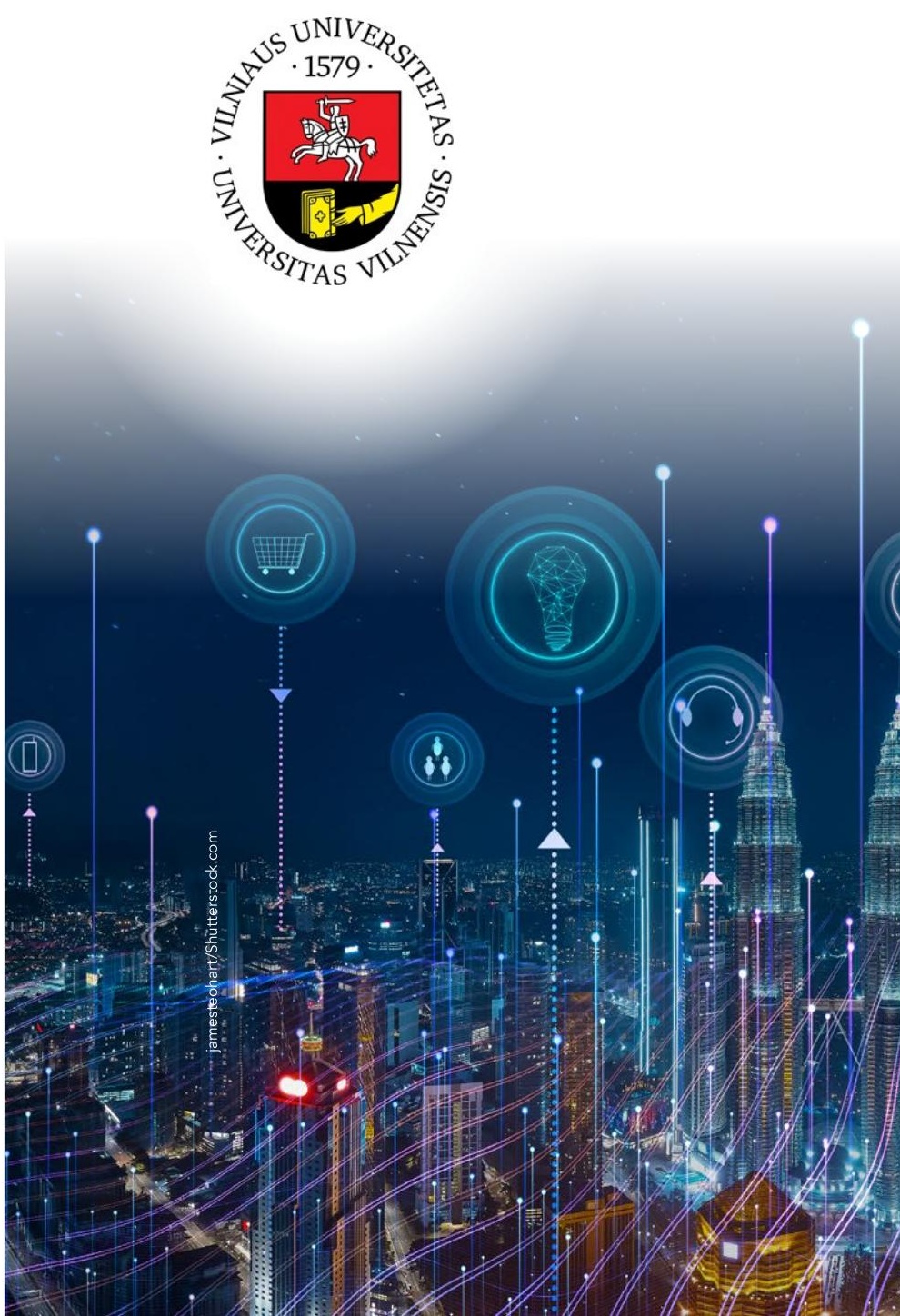

References

Svigaris, Ž, (2021) Instrumentality Versus Awareness: Rethinking the Reverse Side of Artificial Intelligence. In: Rezaei N, (eds) Integrated Science, 1, 301-319. Springer, Cham, doi. org/10.1007/978-3-030-65273-9, 15

\section{Personal Response}

\section{What initially sparked your interest in the paradox of} instrumentality?

II The modern global world has crossed a certain threshold showing that the era of materialism is coming coincidentally with the sunset of the materialistic age is not accidental. It is just another evident sign confirming the limit of its potential. As the materialistic world exhausts its attractiveness, humanity is witnessing the signs of a new type of consciousness and the beginning of a new civilisation. After leaving the material world to technological tools such as artificial intelligence, the consumerism-based mentality can step aside, and culture
can turn to the unexplored, rich psychical qualities of humanity, such as sympathy, empathy and integral balance. This life-affirming and self-sustaining stage of culture via integral consciousness can unfold profound awareness, which was developed for centuries in differen vital part of modern education.

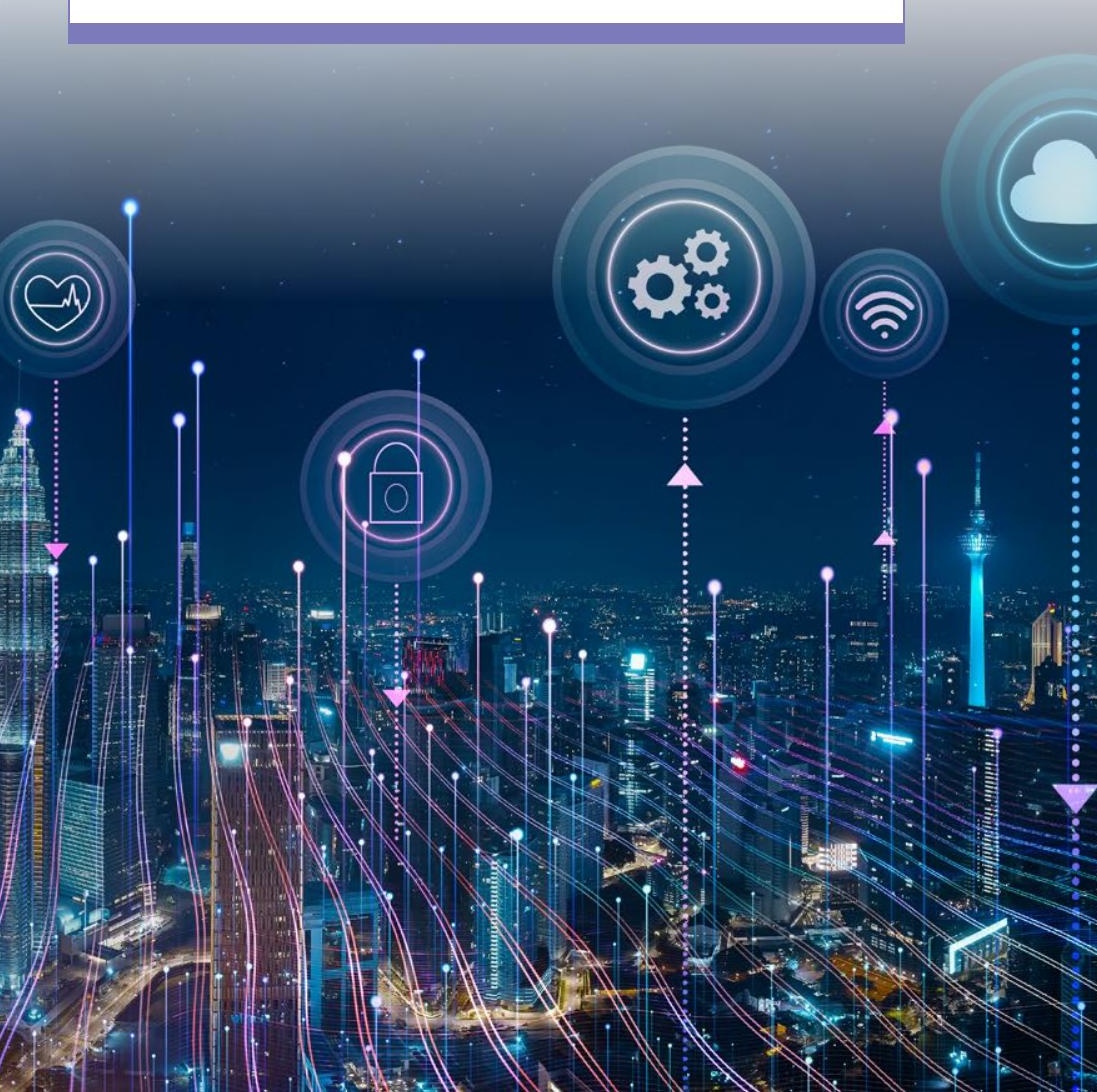

